\title{
Photonic Crystal Fibers Design using a Multicriteria Based Software
}

\author{
I. Sassi ${ }^{1}$, N. Belacel ${ }^{2}$, Y.Bouslimani ${ }^{1}$ and H. Hamam ${ }^{1}$ \\ ${ }^{1}$ Electrical Engineering Department, University of Moncton, Moncton, NB \\ ${ }^{2}$ NRC Institute for Information Technology, Moncton, NB \\ Sassii@umoncton.ca
}

\begin{abstract}
The single-mode optical fiber used currently in communication systems starts showing many limitations especially for the high rates. Several physical phenomena related to the optical propagation are the cause of these limitations. The use of photonic crystal fibers (PCF) makes it possible to control most of these phenomena. In this paper, a multicriteria method is used for the design of the photonic crystal fiber with the user-defined optical proprieties. This method combines the deductive and the inductive learning and it is introduced for the first time in the field of optical fibers. This multicriteria method proves to be a powerful tool for the PCF fibers design.
\end{abstract}

\section{Introduction}

In recent years there has been a major development in optical communications and a new generation of fibers was introduced. These fibers, called photonic Crystal Fibers (PCF) have an unusual propagation properties. In these fibers, the cladding is distinguished from the core only by the presence of air hole what gives him an index of refraction average lower than that of the core. For example, it is possible to produce PCF with very high birefringence [1] , to obtain fibers with very flat near zero chromatic dispersion curve on a large wavelength range [2][3], or to get a single-mode fiber with unusual dispersion regime in the visible wavelength domain [4][5].

The optical properties are related to the fiber design, specifically, the pitch $(\Lambda)$ of the periodic array, the holes diameter $(\mathrm{d})$ and the number $(\mathrm{N})$ of rings around the core. One of these fibers the M-TIR fibers, in this type of fiber the wide choice in the geometrical parameters and the large refractive index difference between air and silica enable much greater flexibility in the design of the dispersion of the guide. Consequently, the chromatic dispersion of M-TIR fibers can be controlled by a suitable choice of the parameters $d$ and $\Lambda[6]$.
Many applications such as telecommunications, supercontinuum generation or parametric amplification require an accurate control of the chromatic dispersion. It is well known that chromatic dispersion can be controlled by adjusting the parameters $\mathrm{d}$ and $\Lambda$ [2].

In this paper, the geometrical parameters are used to design PCF fibers. The optimization of PCF design is often complicated owing to the fact that the optical parameters do not habitually vary in a simple manner with the geometrical parameters of the PCF fiber. This complexity increases exponentially with the numbers of variables of the problems $(\Lambda, N, d \ldots)$ and with the number of properties that have to be considered (chromatic dispersion, confinement losses etc...). The design optimization is principally performed by experiment and test approach. The designer and the computer have to interact regularly with the results of the calculation to design a new type of fiber but this is a time consuming approach.

In this work we apply the multicriteria method to classify PCF and to design the fiber structure. This method contributes to determine the PCF structure starting from the optical characteristic required for a given application. It helps us to overcome some difficulties encountered when data (geometrical and optical proprieties) are expressed in different units. Genetic algorithm method to optimize the PCF structure has been proposed in the literature [8] but multicriteria approach is used for the first time to design PCF fibers and to diagnosis the optical proprieties of these fibers.

The aims of the present study were: (1) to apply the new fuzzy multicriteria assignment method and (2) to design photonic crystal fibers. The present paper is organized as follows: Section 2 presents a general study of the multicriteria methods and the different stages of the PROAFTN method. Section 3 describes the modeling PCF methodology. Section 4 is devoted to the results of the proposed method 


\section{Multicriteria Decision Analysis}

\subsection{Multicriteria methods}

Multicriteria methods have been first introduced by Roy in 1985 [9] and they are now applied in many fields as environment, finances, production, localization, (Vincke, 1987; Roy and Bouyssou, 1993; Pomerol and Al, 1993; Mayster and Al, 1994).

These methods are divided into two groups. The first one includes the automatic classification methods called clustering methods, based on the notion of unsupervised learning, and consists in assembling individuals in restricted classes so that all objects of the same class are less dispersed [10]. The second one comprises the assignment methods, which are based on the notion of supervised learning and employ a set of examples belonging to well-known classes.

In this paper, we apply the PROAFTN method one of the second type of multicriteria method. This method formulated the problem in terms of assigning objects to one or several classes by examining the intrinsic value of each object and by referring to preexisting rules. The problem can be considered as a supervised learning problem. So, PROAFTN method is a multicriteria assignment method based on the preference relational system described by Roy [11] and Vincke [12]. This method employs a comparison between the alternatives through the scores of different criteria. So, it avoids resorting to distance and allows us to use qualitative and /or quantitative criteria. Moreover, it helps us to overcome some difficulties encountered when data are expressed in different units.

\subsection{PROAFTN method}

In this section, we present the PROAFTN method developed by N.Belacel [13], which is able to resolve the multicriteria assignment problems in the nominal sorting problematic. In order to assign the fuzzy belonging degree of action a to each category, PROAFTN determines the fuzzy indifference relations by generalizing concordance and discordance indices used in the ELECTRE III method [9]. The data and notations used by PROAFTN are:

A set of objects or actions to assign to different categories,

$\mathrm{F}=\left\{g_{1} ; g_{2} ; \ldots ; g_{n}\right\}$ set of $\mathrm{n}$ criteria or attributes

$\Omega \quad$ set of k categories or classes such as $\Omega=\left\{C^{1} ; \ldots ; C^{k}\right\}, \mathrm{k} \geq 2$
$B^{h}$ prototype set of $h$ th category, where $B^{h}=\left\{b_{i}^{h} \mid h=1, \ldots, k, i=1, \ldots, L_{h}\right\}$

With $b_{i}^{h}$ designating the $i$ th prototypes of the $h$ th category

$B$ set of all prototypes, such as $\mathrm{B}=\coprod_{h=1}^{k} B^{\text {n }}$

(the set $B$ will be finite and non-empty)

$A$ set of actions $A$ and $B$ such as $A=A \cup B$

The score of actions are evaluated on the criteria set $F$, such as:

$\forall \mathrm{a} \in A$, we have

$$
\begin{aligned}
& g(a)=\left\{g_{1}(a), g_{2}(a), \ldots, g_{2}(a)\right\}, \\
& \forall b_{i}^{h} \in B^{h}, \text { we have } \\
& g\left(b_{i}^{h}\right)=\left\{g_{1}\left(b_{1}^{h}\right), g_{2}\left(b_{1}^{h}\right), \ldots, g_{2}\left(b_{i}^{h}\right)\right\}
\end{aligned}
$$

Where $h$ varies from 1 to $k$ and $i$ varies from 1 to $L_{h}$.

We note 'the action a is assigned to category $c^{\text {h }}$, by a $\in C^{\text {h }}$.

The PROAFTN method uses a synthesis outranking approach and processes and determines the partial indifference relations. Usually, the prototype scores are given by intervals, so for each criterion $g_{j}$, it is associate to each prototype $b_{i}^{h}$ the

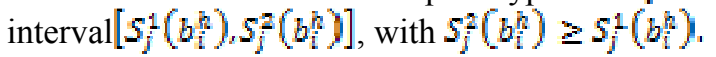

Formally, three comparative situations between the action $a$ and prototype $b_{i}^{a}$ according to criterion $g_{j}$ are obtained using the two discrimination thresholds:

- If $S_{j}^{1}\left(b_{i}^{h}\right) \leq g_{j}(a) \leq S_{j}^{2}\left(b_{i}^{h}\right)$, then a is clearly indifferent to $b_{i}^{\text {R }}$.

- If $\left[g_{j}(\alpha) \leq 5_{j}^{1}\left(b_{i}^{\bar{h}}\right)-d_{j}^{-}\left(b_{i}^{k}\right)\right] \quad$ or $\left[g_{j}(a) \geq S_{j}^{2}\left(b_{i}^{\hat{h}}\right)-d_{j}^{+}\left(b_{i}^{h}\right)\right]$, then a is not indifferent to $b_{i}^{\text {f }}$.

- If $\left[S_{j}^{2}\left(b_{i}^{h}\right)-a_{j}\left(b_{i}^{k}\right)<g_{j}(a)<S_{j}^{1}\left(b_{i}^{\hat{i}}\right)\right]$ or $\left[s_{j}^{2}\left(b_{h}\right)<g_{j}(a)<s_{j}\left(b_{h}\right)-\alpha_{j}^{+}(b \hat{h})\right]$, then there is a weak indifference between a and $b_{l}^{\text {in }}$. $d_{j}\left(b_{h}\right)$ and $d_{j}\left(b_{h}\right)$ are the discrimination thresholds.

Based on argumentation of discrimination thresholds, the partial indifference index $C_{j}\left(a, b_{i}^{\text {h }}\right)$ represents the degree of validity of the three previous situations and verifies the following properties:

$$
\begin{aligned}
& C_{j}\left(a, b_{i}^{h}\right)=1 \leftrightarrow s_{j}^{1}\left(b_{i}^{h}\right) \leq g_{i}(a) \leq s_{j}^{2}\left(b_{i}^{h}\right), \\
& 0<c_{j}\left(a, b_{i}^{h}\right)<1 \leftrightarrow \\
& s_{j}^{1}\left(b_{i}^{h}\right)-d_{j}\left(b_{i}^{h}\right)<g_{j}(a)<s_{j}^{1}\left(b_{i}^{h}\right), \\
& S_{j}^{2}\left(b_{i}^{h}\right)<g_{j}(a)<S_{j}^{2}\left(b_{i}^{h}\right)-d_{j}^{+}\left(b_{i}^{h}\right), \\
& C_{j}\left(a, b_{i}^{h}\right)=0 \leftrightarrow g_{j}(a) \leq s_{j}^{1}\left(b_{i}^{h}\right)-d_{j}^{-}\left(b_{i}^{h}\right), \\
& , g_{j}(a) \geq s_{j}^{2}\left(b_{i}^{h}\right)+d_{j}^{+}\left(b_{i}^{h}\right)
\end{aligned}
$$

The index $C_{j}\left(a, b_{i}^{n}\right)$ is represented between the value $S_{j}^{1}\left(b_{i}^{h}\right)-d_{i}^{-}\left(b_{i}^{h}\right)$ and the $S_{j}^{1}\left(b_{i}^{h}\right)$ on one hand, and 
$S_{f}^{1}\left(b_{i}^{h}\right)$ on one hand, and $S_{f}^{2}\left(b_{i}^{h}\right)$ and $s^{2}(b)+d_{j}^{+}(b h)$ on the other hand, through the linear interpolation function (Figure 1).

From Figure 1, the value of $C_{j}(a, b)$ is determined as follows:

$C_{j}\left(a, b_{i}^{h}\right)=\min \left\{C_{j}^{-}\left(a, b_{i}^{h}\right), C_{j}^{+}\left(a, b_{i}^{h}\right)\right\}$, where

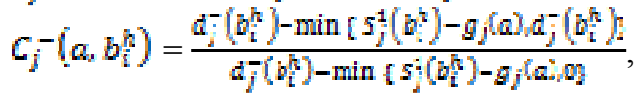

$C_{j}^{+}\left(a, b_{i}^{h}\right)=\frac{d_{j}^{+}\left(b_{i}^{h}\right)-\min \left(g_{j}(a)-s_{j}^{2}\left(b_{i}^{h}\right) d d_{j}^{+}\left(b_{i}^{h}\right)\right.}{d_{j}^{f}\left(b_{i}^{h}\right)-\min \left[g_{j}(a)-s_{j}^{2}\left(b_{i}^{h}\right) \omega q\right.}$.

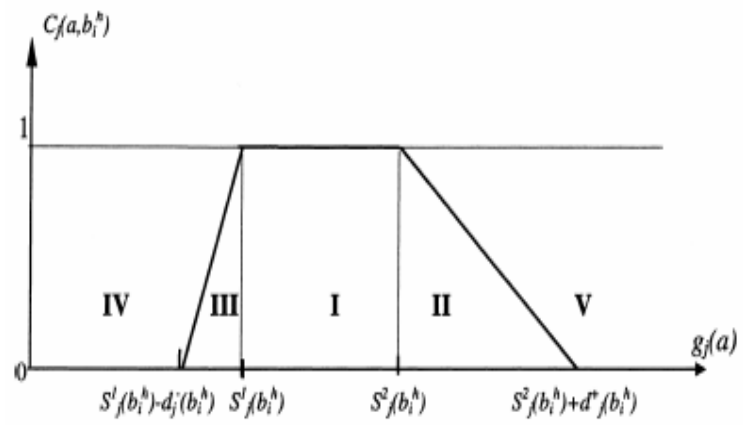

Figure 1. Graphic representation of the partial concordance index.

Since the partial concordance indices, the comprehensive concordance index is defined in the following way, $C_{I}\left(a, b_{i}^{n}\right)=\sum_{j=0}^{n}\left(w_{j}^{h} * C_{j}\left(a, b_{i}^{n}\right)\right)$ where $w_{j}^{h}, \mathrm{j}=1, \ldots, \mathrm{n}$ and $\mathrm{h}=1, \ldots, \mathrm{k}$, are positives weights adding to one and reflecting the intrinsic relative importance attached by a decision maker to a criterion $g_{j}$. The PROAFTN method determines also the partial discordance index $D_{j}\left(a, b_{i}^{n}\right)$. This index is represented between the value $S_{j}^{1}\left(b_{i}^{h}\right)-v_{j}^{-}\left(b_{i}^{h}\right)$ and the $S_{j}^{1}\left(b_{i}^{h}\right)-d_{j}^{-}\left(b_{i}^{h}\right)$ on one hand and $S_{j}^{2}\left(b_{i}^{h}\right)-d_{j}^{+}\left(b_{i}^{h}\right)$ and $S_{j}^{2}\left(b_{i}^{h}\right)-v_{j}^{+}\left(b_{i}^{h}\right)$ on the other hand, by the linear interpolation function (Figure 2). $v_{j}^{-}\left(b_{i}^{h}\right)$ and $v_{j}^{+}\left(b_{i}^{h}\right)$ are the veto thresholds. From Figure 2, the discordance is deduced as follows:

$D_{j}\left(a, b_{i}^{h}\right)=\max \left\{D_{j}^{-}\left(a, b_{i}^{h}\right), D_{j}^{+}\left(a, b_{i}^{h}\right)\right\}$, where $D_{j}^{-}\left(a, b_{i}^{h}\right)=\frac{g_{j}(a)-\max \left[g_{j}\left(a_{i}, s_{j}^{1}\left(b_{i}^{h}\right)-d_{j}\left(b_{i}^{h}\right)\right)\right.}{d_{j}\left(b_{i}^{h}\right)-\max \left(s_{j}^{1}\left(b_{i}^{h}\right)-g_{j}\left(a_{j} v_{j}^{-}\left(b_{i}^{h}\right) j\right.\right.}$,

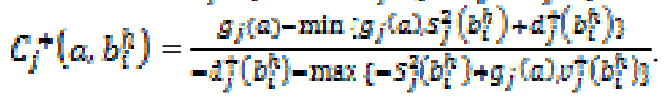

From these partial discordance indices, the comprehensive discordance index is defined in the following way:

$D_{2}(a, b)=1-\prod_{j=1}^{n}\left(1-D_{2}(a, b h)^{w_{j}^{h}}\right.$

With theses indices, we can assign an action to predefined categories by the analysis of the fuzzy relations.

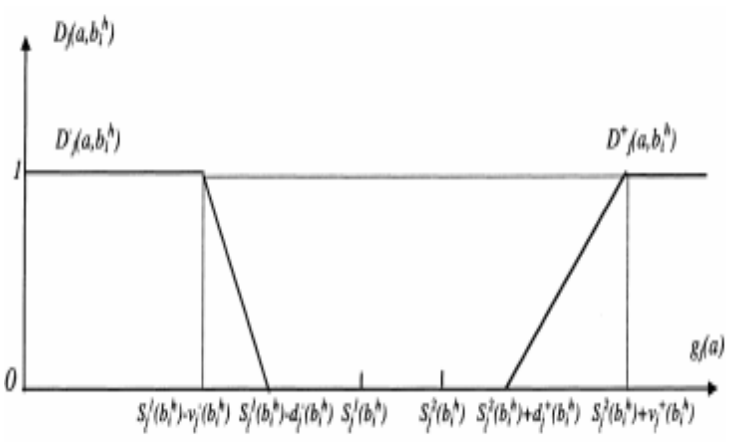

Figure 2. Graphic representation of the partial discordance index.

\section{Photonic Crystal Fibers Modeling}

In this section, we explain our reasoning to design the photonic crystal fibers (PCF). In order to design a PCF fiber, we start by building database (training and test).We have elaborated a training dataset with certain photonic crystal fibers. Then, we have reconstructed a simulated fiber with a known geometrical characteristics (core diameter, distance between two holes, cladding dimension...) and unknown optical characteristics. After that, we applied the multicriteria classification method in order to obtain the optical characteristics. In fact, the classification procedure POAFTN is based on the fuzzy indifference relation between a fiber to model and the training fiber data. By means of the assignment decision, we can have an idea about the optical characteristics. To model a photonic crystal fiber, we start from the structure of the fiber (the geometrical proprieties), we can calculate the optical properties as the chromatic dispersion .With the optimization of these proprieties, we control the structure of the PCF fiber that we want to design, as shown in Figure 3. 


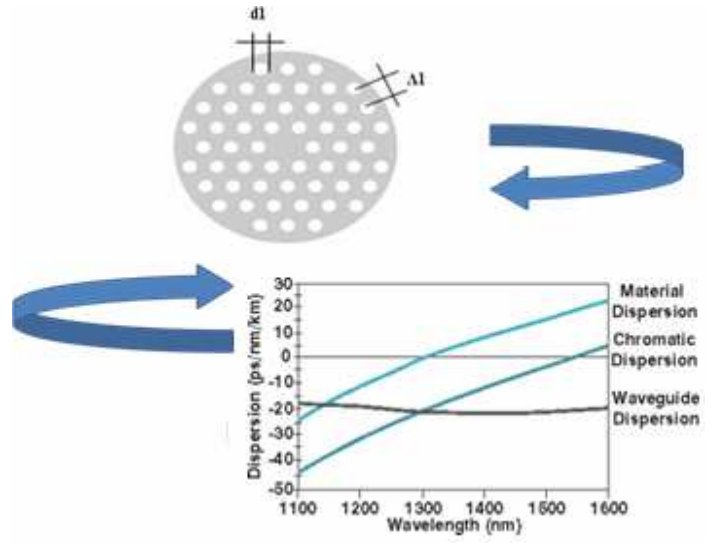

Figure 3. PCF modeling

As stated above, the photonic crystal fiber model can be finalized with the simulation. To model a PCF fiber, we proceed by the following steps. We consider a simulated fiber with defined geometrical characteristic and undefined optical characteristic using the training dataset and the multicriteria procedure; we can classify this fiber to a category of photonic crystal fibers (for example the large mode PCF fiber...).We can evaluate the fuzzy indifference relation between a fiber to design and the prototypes and through assignment decision; so we can have an idea about the fiber model.

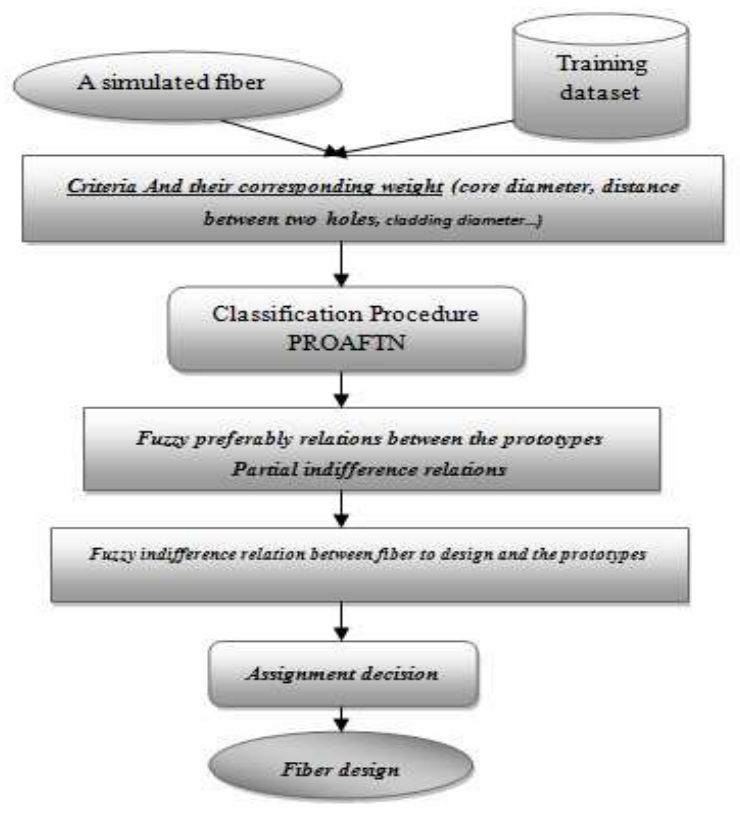

Figure 4. Multicriteria classifier

\section{Results}

In order to design photonic crystal fibers, we have developed software based on a multicriteria classification method. This tool makes it possible to classify a fiber to any categories of photonic crystals fibers (large mode fibers, maintaining polarization fibers...).Aiming at analyzing optical characteristics of these fibers, we defined and measured the fuzzy indifference relations between a fiber to model and the proptypes.The figure 5 shows the interface of the developed tool.

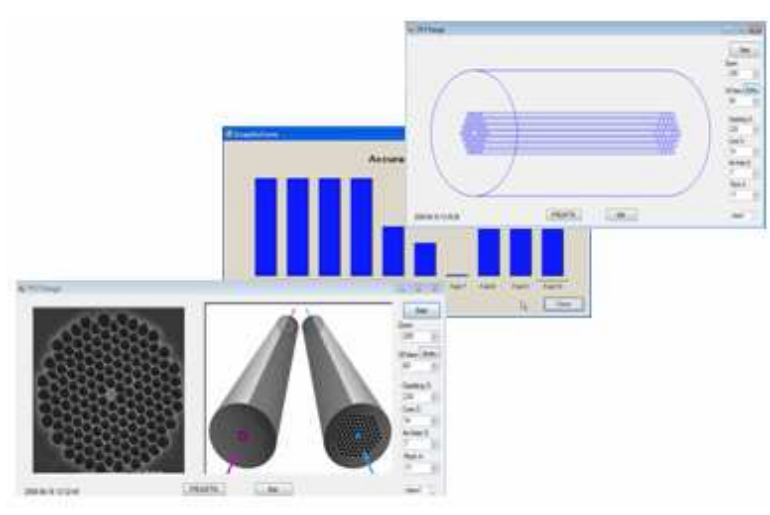

Figure 5. PCF Design Software

We have tested the validity of this method. The advantage of the photonic crystal fiber HC-19-532 (see Figure 6) a low loss rate transmission. This fiber is classified to the class of low loss PCF fiber with $95 \%$ of accuracy. So, we have confirmed that this fiber is a member of the category of low loss fiber.
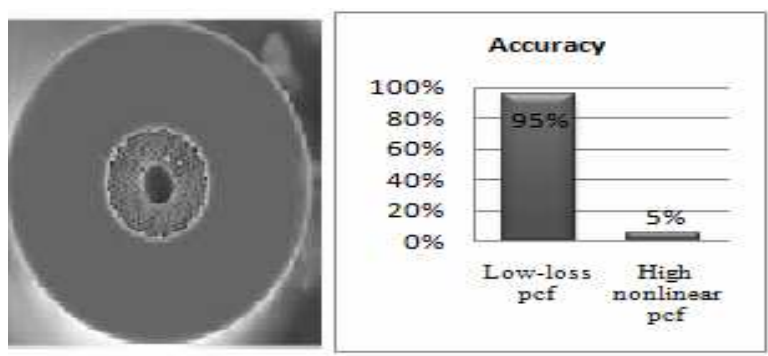

Figure 6. PROAFTN analysis for HC-19-532 


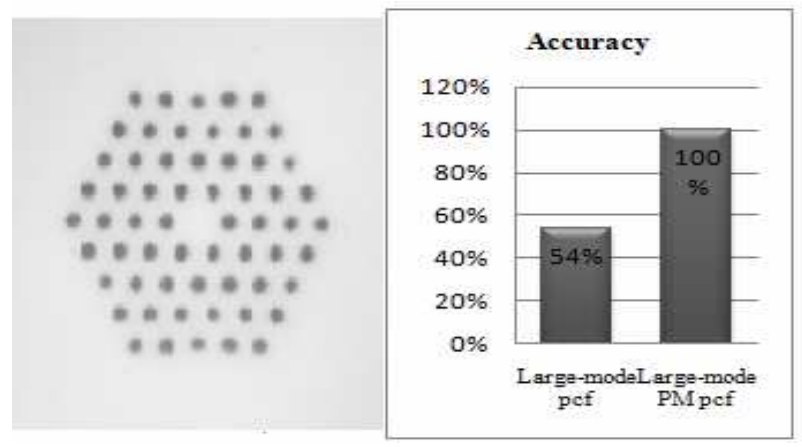

Figure 7. PROAFTN Analysis for LMA-PM-5

Figure 7 shows an other example of the tested PCF fibers. This large core polarization maintaining photonic crystal fiber is optimized for single-mode operation in the UV to $800 \mathrm{~nm}$ wavelength range and it is an endlessly single mode fiber. With PROAFTN analysis, we have approved that this fiber is large mode polarization maintaining fiber. For this type of fiber, we obtained a high membership scores to the large mode PM PCF, in the same time it is a member of the large mode PCF fiber. So, for the same fiber, we can have two characteristics or more of the classified fiber and this fiber can be assigned to two classes.

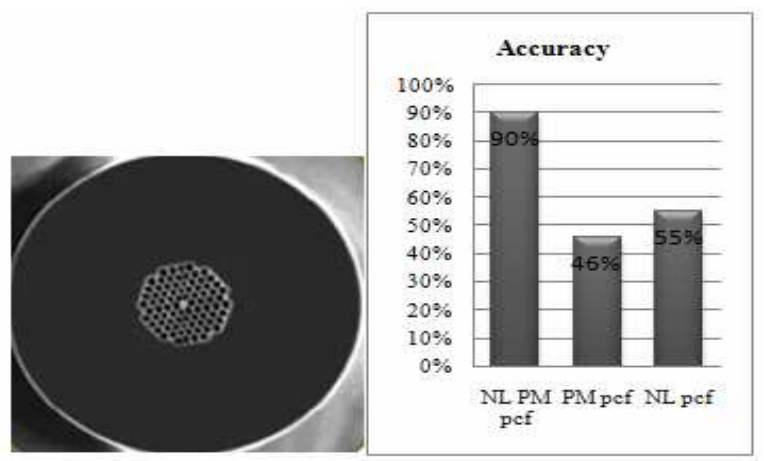

Figure 8. PROAFTN Analysis for NL-PM-750

The obtained results for the NL-PM-750 confirm that this fiber is a high nonlinear polarization maintaining fiber with a $90 \%$ rate of accuracy. So, the developed software is able to classify photonic crystal fibers and to model the fiber structure with the appropriate optical proprieties aiming at designing a PCF fiber.

\section{Conclusions}

In the current study, we have presented a novel approach for the photonic crystal fibers design. Our approach is based on a multicriteria method. Each category of PCFs is defined by a set of reference patterns. The fuzzy modeling eliminates the problem of strict thresholding (and the need for physical description), while the multicriteria method employs a comparison between fibers through the use of values in different criteria. The application of the PROAFTN method was successful and modeled suitably the geometrical and the optical characteristics of PCF fibers. On the other hand, the employment of the developed software makes it possible to test the adopted method. Furthermore, the developed PCF fiber database contributed significantly to the proper training and testing of the whole system.

\section{Acknowledgments}

The authors wish to thank the Natural Sciences and Engineering Research Council of Canada for the financial support.

\section{References}

[1] A. O. Blanch, J. C. Knight, W. J. Wadsworth, J. Arriaga, B. J. Mangan, T. A. Birks, and P. St. J. Russell, "Highly birefringent photonic crystal fibers," Opt. Lett. 25, 2000.

[2] J.C.Knight, W.H.Reeves, and P.St.J.Russell, "Demonstration of ultra-flattened dispersion in photonic crystal fibers," Opt. Express 10, 2002.

[3] A. Ferrando, E. Silvestre, J.J. Miret, and P. Andrés, "Nearly zero ultraflattened dispersion in photonic crystal fibers," Opt. Lett. 25, 2000.

[4] D. Mogilevtsev, T. A. Birks, and P. St. J. Russell, "Group-velocity dispersion in photonic crystal fibers," Opt. Lett 23,1998 .

[5] J. K. Ranka, R. S. Windeler, and A. J. Stentz, "Visible continuum generation in air-silica microstructure optical fibers with anomalous dispersion at $800 \mathrm{~nm}$," Opt. Lett 25 , 2000.

[6] A. Ferrando, Silvestre E, Miret J J, Monsoriu J A, Andres M V and Russell P St J " Designing a photonic crystal fibre with flattened chromatic dispersion " Electron. Lett. 35 325, 1999.

[8] Kerrinckx Emmanuel, Laurent Bigot, Marc Douay, Yves Quiquempois, 2004, "Photonic crystal fiber design by means of a genetic algorithm," OSA 9, Vol. $12,2004$. 
[9] Roy .B, ELECTRE III: Un algorithme de classement fondée sur une représentation floue des préférences en présence de critères multiples, Cahiers du CERO 20, 1978, pp. 3-24.

[10] G. Celeux, E. Diday, G. Govaert et al., Classification automatique de données environnement statistique et informatique, Dunod Informatique, France, 1989.

[11] B. Roy, Multicriteria Methodology for Decision Aiding, Kluwer Academic Publishers, Dordrecht, 1996.
[12] Ph. Vincke, Multicriteria Decision Aid, Wiley, New York, 1992.

[13] N. Belacel, "Multicriteria assignment method PROAFTN: Methodology and medical application", European Journal of Operational Research, Elsevier, 2000, pp. $175-183$. 